\title{
Altered interactions of tryptophan metabolites in first- episode neuroleptic-naive patients with schizophrenia
}

\author{
JK Yao ${ }^{1,2,3}$, GG Dougherty Jr', ${ }^{1,2}$ RD Reddy ${ }^{1,2}$, MS Keshavan²,4,5, DM Montrose ${ }^{2}$, WR Matson ${ }^{6}$, \\ S Rozen ${ }^{7}$, RR Krishnan ${ }^{8}$, J McEvoy ${ }^{8}$ and R Kaddurah-Daouk ${ }^{8}$ \\ ${ }^{1}$ VA Pittsburgh Healthcare System, Pittsburgh, PA, USA; ${ }^{2}$ Department of Psychiatry, Western Psychiatric Institute \& Clinic, \\ University of Pittsburgh Medical Center, Pittsburgh, PA, USA; ${ }^{3}$ Department of Pharmaceutical Sciences, University of \\ Pittsburgh School of Pharmacy, Pittsburgh, PA, USA; ${ }^{4}$ Department of Psychiatry and Behavioral Neurosciences, Wayne State \\ University, Detroit, MI, USA; ${ }^{5}$ Department of Psychiatry, Beth Israel Deaconess Medical Center and Harvard University, \\ Boston, MA, USA; ${ }^{6}$ Bedford VA Medical Center, Bedford, MA, USA; ${ }^{7}$ Whitehead Institute for Biomedical Research, Cambridge, \\ MA, USA and ${ }^{8}$ Duke University Medical Center, Durham, NC, USA
}

\begin{abstract}
Schizophrenia is characterized by complex and dynamically interacting perturbations in multiple neurochemical systems. In the past, evidence for these alterations has been collected piecemeal, limiting our understanding of the interactions among relevant biological systems. Earlier, both hyper- and hyposerotonemia were variously associated with the longitudinal course of schizophrenia, suggesting a disturbance in the central serotonin (5-hydroxytryptamine (5-HT)) function. Using a targeted electrochemistry-based metabolomics platform, we compared metabolic signatures consisting of 13 plasma tryptophan (Trp) metabolites simultaneously between first-episode neuroleptic-naive patients with schizophrenia (FENNS, $n=25$ ) and healthy controls $(\mathrm{HC}, n=30)$. We also compared these metabolites between FENNS at baseline (BL) and 4 weeks (4w) after antipsychotic treatment. $\mathrm{N}$-acetylserotonin was increased in FENNS-BL compared with $\mathrm{HC}(P=0.0077$, which remained nearly significant after Bonferroni correction). $\mathrm{N}$-acetylserotonin/Trp and melatonin (Mel)/serotonin ratios were higher, and Mel/ $\mathrm{N}$-acetylserotonin ratio was lower in FENNS-BL (all $P$-values $<0.0029$ ), but not after treatment, compared with HC volunteers. All three groups had highly significant correlations between Trp and its metabolites, Mel, kynurenine, 3-hydroxykynurenine and tryptamine. However, in the HC, but in neither of the FENNS groups, serotonin was highly correlated with Trp, Mel, kynurenine or tryptamine, and 5-hydroxyindoleacetic acid (5HIAA) was highly correlated with Trp, Mel, kynurenine or 3-hydroxykynurenine. A significant difference between HC and FENNS-BL was further shown only for the Trp-5HIAA correlation. Thus, some metabolite interactions within the Trp pathway seem to be altered in the FENNS-BL patients. Conversion of serotonin to $\mathrm{N}$-acetylserotonin by serotonin $\mathrm{N}$-acetyltransferase may be upregulated in FENNS patients, possibly related to the observed alteration in Trp-5HIAA correlation. Considering $\mathrm{N}$-acetylserotonin as a potent antioxidant, such increases in $\mathrm{N}$-acetylserotonin might be a compensatory response to increased oxidative stress, implicated in the pathogenesis of schizophrenia.
\end{abstract}

Molecular Psychiatry (2010) 15, 938-953; doi:10.1038/mp.2009.33; published online 28 April 2009

Keywords: Trp; $\mathrm{N}$-acetylserotonin; 5-hydroxyindoleacetic acid; schizophrenia; first-episode neuroleptic naive; targeted metabolomics

\section{Introduction}

Schizophrenia is a remarkably complex disorder with a multitude of behavioral and biological perturbations. Whether these diverse alterations are independent biological processes or a result of a more fundamental pathology has yet to be determined. A multiplicity of theories have been proposed over the

Correspondence: Dr JK Yao, Neurochemistry and Psychopharmacology Laboratory, VA Pittsburgh Healthcare System, Building 13, 151R2-H, 7180 Highland Drive, Pittsburgh, PA 15206, USA. E-mail: jkyao@pitt.edu

Received 29 July 2008; revised 16 February 2009; accepted 2 April 2009; published online 28 April 2009 years that aim to conceptualize the pathological processes inherent to schizophrenia, including altered neurotransmission and signal transduction, neuropeptides, autoimmune dysfunction and many others. ${ }^{1-5}$ Treatment likely modifies these metabolic processes, but which of the many metabolic pathways are modified and of what relevance they are to clinical outcome is a mystery. Thus, the issue at hand, first, is to identify the candidate biological process(es) that are associated with schizophrenia. Such an approach can lead to the identification of specific metabolic pathways that can serve as potential targets for therapeutic monitoring and intervention. We now have the means to address these questions with some degree of confidence. 
Analyzing biological entities as integrated systems of genetic, genomic, protein, metabolite, cellular and pathway events that are in flux and interdependent is a new paradigm. ${ }^{6}$ The application of metabolomics, which demands high-resolution multidimensional separation techniques, will transform the investigation of major psychiatric disorders, such as schizophrenia, depression and bipolar disorders. ${ }^{7-11}$ Research into a small number of neurotransmitter systems-dopaminergic, serotonergic, glutamatergic and adrenergic-has shown up a large number of alterations, putatively associated with the disorders in question. However, the utility of these data in understanding these disorders has remained limited because neurotransmitter systems are linked to each other, to signal transduction systems and to a broad variety of membrane-bound and cellular systems.

Psychoses induced by indole hallucinogenic drugs, for example, lysergic acid diethylamide, have been used as a model in the pathophysiology of schizophrenia. Earlier investigations suggested hyperserotonemia may play an etiological role in schizophrenia. ${ }^{12-15}$ However, hyperserotonemia has not been replicated in other studies. ${ }^{16,17}$ In contrast, 5-hydroxytryptamine (5-HT) deficiency has also been proposed in schizophrenia because of (i) decreased level of 5-hydroxyindoleacetic acid (5HIAA) in the cerebrospinal fluid (CSF); ${ }^{18,19}$ (ii) low level of plasma tryptophan (Trp) ${ }^{20,21}$ and (iii) some clinical improvement after 5-HT precursor or Trp treatment. ${ }^{22,23}$

After the development of atypical antipsychotic drugs that potently block $5-\mathrm{HT}_{2}$ receptors, there was renewed interest in 5-HT for schizophrenia research. Antipsychotic drugs, such as clozapine and risperidone, have unique clinical properties resulting from their pharmacological actions on both dopamine and 5-HT receptors. ${ }^{24,25}$ Thus, 5-HT receptors, which are distributed in the brain regions, may be mediated by neuroleptics to regulate complex behavior processes. It is thus important to further understand how these atypical antipsychotic drugs affect not only the 5-HT pathway but also other linked biochemical pathways.

Tryptophan dysregulation may also be important in the glutamate system. One of the key metabolites in the Trp pathway, kynurenine (Kyn), has been implicated in schizophrenia. ${ }^{26}$ Trp is either transaminated to kynurenate, a glutamate receptor antagonist, or is hydroxylated to 3-hydroxykynurenine (3OHKy), which is further degraded to the excitotoxic $\mathrm{N}$-methyl-D-aspartate agonist, quinolinate. In view of the hypoglutamatergic hypothesis of schizophrenia, ${ }^{27}$ alterations in the kynurenine pathway metabolism have been implicated in schizophrenia.

Utilizing the novel powerful and rapid multidimensional separation and characterization methods, for example, high-pressure liquid chromatography coupled with electrochemical coulometric array detection (LCECA), can lead to revolutionary changes in our understanding at the molecular level..$^{28-30}$ The resolving power of these methods is superior to onedimensional approaches, enabling comprehensive metabolic analyses, particularly in the targeted biochemical pathways. Parallel with these developments has been advances in informatics capable of rigorous qualitative and quantitative analyses allowing the development of exploratory models that will propel biological psychiatric research. In this study, we compared metabolic signatures consisting of 13 Trpdegraded products simultaneously in the plasma between first-episode neuroleptic-naive patients with schizophrenia (FENNS, $n=25$ ) and healthy controls (HC, $n=30$ ), as well as between FENNS at baseline (BL) and 4 weeks (4w) after antipsychotic treatment. Specifically, we examined (a) whether Trp metabolites differ among FENNS-BL, FENNS-4 $\mathrm{w}$ and HC volunteers and (b) whether the intercorrelations between these metabolites, which reflect the integrity of these metabolic pathways, differ among the three groups.

\section{Materials and methods}

\section{Clinical design}

First-episode neuroleptic-naive patients. A total of 25 first episodes of psychosis patients (Table 1) were recruited after they provisionally met DSM-IV criteria for schizophrenia, schizophreniform or schizoaffective disorder based on Structured Clinical Interview for DSM Disorders. The initial diagnostic assessments were made by experienced research clinicians. Initial and follow-up diagnoses were confirmed at a diagnostic conference attended by research faculty and staff, chaired by an experienced psychiatrist (MSK). All patients signed informed consent after full explanation of the study. Blood samples were obtained at BL in patients (FENNS-BL) before the initiation of antipsychotic agents. A second set of blood samples was obtained in the same patient individuals about 4 weeks after treatment (FENNS-4w) with one or more of the following antipsychotic drugs: risperidone $(n=17)$, olanzapine $(n=4)$, quetiapine $(n=2)$, aripiprazole $(n=1)$ and haloperidol $(n=2)$. The number adds up to more than 25 because of polypharmacy.

Normal controls. A total of 30 age-matched HC volunteers (Table 1) were recruited through local advertisement. Volunteers with earlier exposure to antipsychotic agents, a lifetime history of an Axis I disorder, systemic medical illness requiring treatment and neurological disorders were excluded. It is interesting to note that the body weight of both male and female volunteers was significantly higher in a normal control group than in a FENNS group (Table 1).

\section{Sample preparation}

All blood samples were collected in the morning after overnight fasting. Samples were prepared for analysis by extraction in acidified acetonitrile and analyzed by LCECA as described earlier. ${ }^{30-33}$ Briefly, freshly drawn blood with anticoagulant citrate dextrose was 
Table 1 Participants' characteristics

\begin{tabular}{|c|c|c|c|c|}
\hline \multirow[t]{2}{*}{ Demographical features } & \multicolumn{2}{|c|}{ Healthy controls } & \multicolumn{2}{|c|}{ FENNS } \\
\hline & Male & Female & Male & Female \\
\hline Number & 18 & 12 & 19 & 6 \\
\hline Age (years) & $22.5 \pm 4.5$ & $23.2 \pm 4.6$ & $21.4 \pm 5.5$ & $26.3 \pm 10.6$ \\
\hline Educations (years) & $14.3 \pm 3.1$ & $13.9 \pm 2.3$ & $11.8 \pm 2.9$ & $12.3 \pm 4.6$ \\
\hline Weight (lbs) & $185.9 \pm 39.7$ & $140.4 \pm 19.3^{\mathrm{a}}$ & $156.1 \pm 43.5^{\mathrm{b}}$ & $120.0 \pm 19.2^{\mathrm{a}, \mathrm{b}}$ \\
\hline Height (inches) & $70.6 \pm 3.2$ & $64.9 \pm 2.6^{\mathrm{c}}$ & $68.2 \pm 4.1$ & $64.3 \pm 1.7^{\mathrm{c}}$ \\
\hline Body mass index & $26.6 \pm 5.4$ & $23.8 \pm 3.0$ & $23.9 \pm 4.8$ & $20.7 \pm 3.0$ \\
\hline
\end{tabular}

Abbreviations: FENNS, First-episode neuroleptic-naive patients with schizophrenia.

${ }^{\mathrm{a}} P=0.0003$ ( $t$-test, gender covariate effect on weight).

${ }^{\mathrm{b}} P=0.006$ ( $t$-test group effect on weight).

${ }^{\mathrm{c}} P<0.0001$ ( $t$-test, gender covariate effect on height).

centrifuged at $750 \mathrm{~g}$ for $7 \mathrm{~min}$ to remove red blood cells and stored at $-80{ }^{\circ} \mathrm{C}$ in a freezer. An aliquot $(250 \mu \mathrm{l})$ of a stored sample was mixed with $1 \mathrm{ml}$ of acetonitrile $/ 0.4 \%$ acetic acid at $-25{ }^{\circ} \mathrm{C}$ and vortexed for 45-60 s; then the temperature was brought to $-15^{\circ} \mathrm{C}$ in a cold block, and vortexed again for $30-45 \mathrm{~s}$. The samples were centrifuged for $15 \mathrm{~min}$ at $12000 \mathrm{~g}$ at $4{ }^{\circ} \mathrm{C}$. In total, $1 \mathrm{ml}$ of the resulting supernate was transferred to a $2 \mathrm{ml}$ screw top vial and evaporated under vacuum. It is critical that the vacuum is sufficient to freeze the sample during this step. The sample was reconstituted in $200 \mu \mathrm{l}$ of mobile phase A and $100 \mu \mathrm{l}$ were loaded onto two autosampler vials, one of which was archived at $-80^{\circ} \mathrm{C}$. Profiles are stable in acetonitrile extract, dried extract and mobile phase-diluted extract.

During sample preparation, pools were created from equal volumes of aliquots of all samples. All assays were run in sequences that include 10 samples, authentic reference standard mixtures of 80 known compounds, pools of all samples and duplicate preparations of the same sample. Duplicates are spaced at short and long intervals through the run to reflect the performance of the total database. Run orders of all samples in this study were randomized. The sequences minimized possible analytical artifacts during further data processing. Pools and duplicates were used to access the precision of the entire data set. In addition, the pools were used as references for time normalization (stretching). A practical advantage of LCECA for this study is the relative freedom from maintenance events. This is important for the generation of consistent databases from large numbers of samples over extended time periods. In our earlier work we have run LCECA continuously for $24 \mathrm{~h}$ per day over 6 months.

High-pressure liquid chromatography coupled with electrochemical coulometric array detection

The LCECA method used in this study has been described earlier ${ }^{29,30}$ and applied to studies of dietary restriction in a rat model, ${ }^{31}$ lower motor neuron disease $^{32}$ and Parkinson's disease ${ }^{33}$ Briefly, the liquid chromatographic method employs an A mobile phase $\left(10.3 \mathrm{gl}^{-1}\right.$ sodium pentane sulfonate, $5 \mathrm{ml} \mathrm{l}^{-1}$ glacial acetic acid) and a B mobile phase (methanol/ acetonitrile/isopropanol 8/1/1, $8 \mathrm{gl}^{-1}$ lithium acetate, $20 \mathrm{ml} \mathrm{l}^{-1}$ glacial acetic acid). A gradient is run from $100 \%$ A to $100 \%$ B over $120 \mathrm{~min}$. The electrochemical array of 16 series coulometric detectors is set from 0 to $900 \mathrm{mv}$ in equal $60 \mathrm{mv}$ increments from detector 1 to 16. In this mode a compound passing through a coulometric electrode is oxidized by $100 \%$ of the thermodynamically possible amount. This results in a characteristic signature for a compound expressed as a ratio on sequential electrodes. This ratio provides a high degree of qualitative certainty, which can be set for any particular study. ${ }^{30}$ The gradient and detector conditions typically provide responses at the $500 \mathrm{pg} \mathrm{ml}^{-1}$ level ( $5 \mathrm{pg}$ on column) for ca 1500 2000 compounds in biological samples. In comparison with mass spectrometry (MS) a specific thermodynamically determined response ratio and retention time in an LCECA method does not carry as much qualitative certainty as an accurate mass peak or fragmentation pattern in MS/MS. However, in comparison with MS for the classes of compounds measurable on LCECA the sensitivity of ca $500 \mathrm{pg} \mathrm{ml}^{-1}$ is typically one to two orders of magnitude lower than can be achieved with MS. As an example, we conducted a study directed at identifying metabolites implied by the presence of multiple responses in an LCECA method following Huntington's disease patients treated with phenyl butyrate. ${ }^{34}$ The LCECA method employed $40 \mu \mathrm{l}$ of plasma. It was necessary to concentrate and fractionate $4 \mathrm{ml}$ of plasma to obtain sufficient material for qualitative identification in a parallel LCECA/LCMS system. The LCECA method with $100 \%$ efficient electron transfer also has an inherent quantitative control based on the integration of the total coulombs of the peaks ${ }^{34}$ and the calculation of quantity by Faraday's law. Thus, it is independent of such factors as variations in ionization efficiency as a result, for instance, of column bleed.

Inter-laboratory/inter-method comparisons are a field in and of themselves. Well-designed studies 


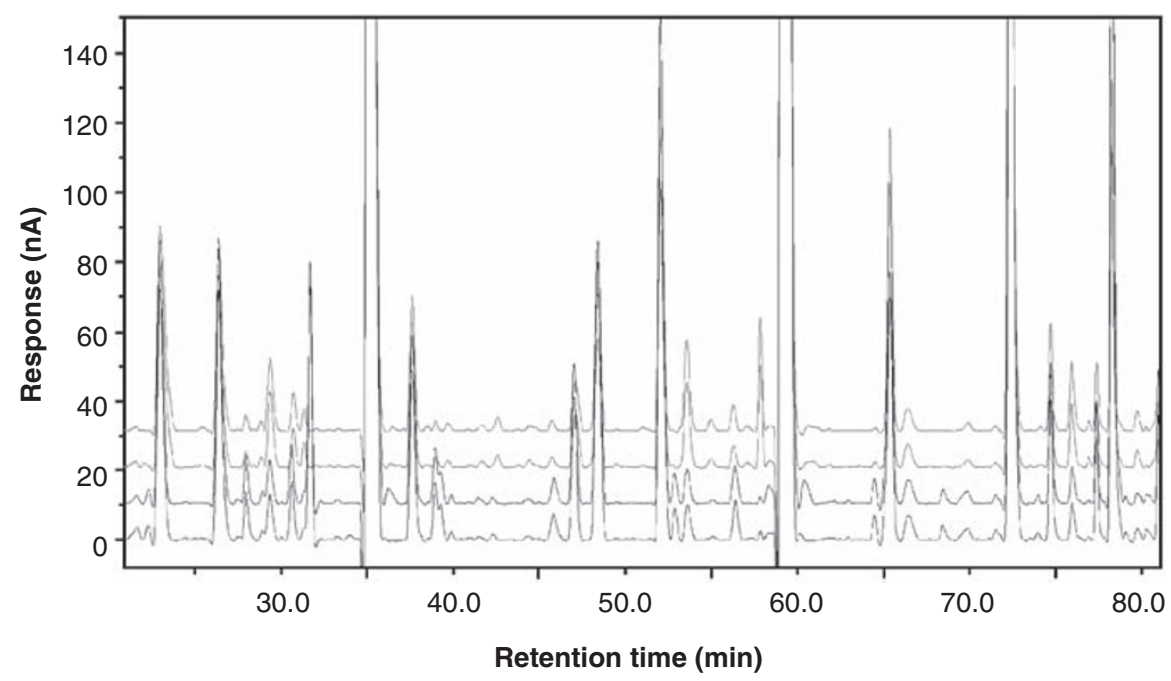

Figure 1 The time and response stability of the high-pressure liquid chromatography coupled with electrochemical coulometric array detection (LCECA) platform, showing the use of time normalization protocols in data reduction and stability of electrochemical sensors response. Two upper traces-plasma pools analyzed 2 months apart. Two lower traces-duplicates of a sample from patient with Parkinson's disease analyzed 4 months apart. Channel 12 of LCECA chromatogram is shown.

are highly expensive and have to take into account standards, preparative methods, sample splitting techniques and so on, as well as the instrumentation and parameters of instrument usage. Initial efforts are frequently discouraging. As an example the initial round of the multi-center/method ESCOT study for 8 hydroxy 2 'deoxyguanosine measurements initially returned values differing by a factor of 1000 with the higher values resulting from source artifacts in a gas chromatography-mass spectrometry (GCMS) method.

Enzyme-linked immunosorbent assay techniques for this same analyte in urine are typically comparable to electrochemical methods for controls and standards but a factor of $2-5$ higher in various disorders, whereas GCMS techniques for this analyte in CSF have been reported as 10000 times higher compared with electrochemical techniques.

\section{Data reduction and analysis}

All chromatograms in the study were backgroundcorrected to eliminate the base line drift inherent in gradient profiles. By controlling the analytical conditions, the location of any particular peak in a 16channel 110-min chromatogram was held within \pm (5-30) s through the study. Background-corrected files were then sequentially time-normalized against a single pool in the middle of the study sequence. A two-step stretching protocol with a multitude of peaks was used. First, proprietary software (ESA, CEAS 512 ESA, Inc., Chelmsford, MA, USA) was used to align 15-20 major peaks in the chromatogram and interpolate the positions between them. Then, an additional 20-25 smaller peaks present in most samples were selected from the derivative file and those were realigned, keeping the major peaks in the same position. Selected peaks were aligned within $\pm 0.5 \mathrm{~s}$ and non-selected peaks within $\pm(1-1.5) \mathrm{s}$ over the entire $110 \mathrm{~min}$ assay. An example of two pools analyzed 2 months apart, and a duplicate sample from a subject analyzed at the beginning and at the end of the study 4 months later, is shown in Figure 1.

Data were exported in three formats. First, all responses matching the retention and electrochemical signature of compounds in the reference standard were exported in concentration units of $\mathrm{ng} \mathrm{ml}^{-1}$. Second, all responses matching resolved peaks in the pool of all samples were exported in terms of their relative response to the pool value. The concentrations of these were subsequently estimated by the total coulombs in the peak assuming a molecular weight of 200 and a two-electron charge transfer.

Third, in addition to determining the concentration levels of peaks against standards and pools, we exported the analytical information for all samples in digital format (digital maps). ${ }^{34}$ Using complete digital output served two purposes: (i) capture of all analytical information for the following data analysis and (ii) avoiding possible artifacts introduced by peak-finding algorithms. The number of variables in the digital maps depends on the resolution set during the data export. In this study, the resolution was set at $1.5 \mathrm{~s}$ and the number of data points (variables, defined as the signal at a given time on a given channel) obtained from one sample, using our current LCECA approach, was 66000 . It is important to note that the number of variables in digital maps is not equivalent to the number of analytes because an individual analyte is represented by more than one variable. Depending on the concentration of an analyte and on its separation across the electrochemical array, the number of variables characterizing an analyte could be between 10 and 100. In the consolidated files of a study all variables were aligned in a spreadsheet for data analysis with each column representing a single subject (sample) organized by time from channel 1 to 16. Each row in a spreadsheet represents the 
response of a compound (variable) at a specific time and channel for all samples. This approach avoids artifacts in data reduction and protects against overfitting in the data analysis. Before data analysis, rows in the digital maps for which all values were negative or $<30 \mathrm{pA}$ (noise level of the analytical method), for all samples were eliminated. The data were analyzed by conventional statistical methods and by partial least squares discriminant analysis. ${ }^{35}$ After finding the variables differentiating the groups (for example, schizophrenic vs control volunteers), we sorted the variables by retention time and channel. This step allowed the isolation of 'peak clusters' (that is, all digital map variables characterizing one specific analyte), which, in turn, provides an identification of specific markers. Then the most significant variables in the digital maps were used to identify the location of the actual marker peaks within the chromatograms.

\section{Analysis of Trp metabolism}

Generally, a metabolic pathway consists of sequential biochemical reactions that generate various products from a set of precursors. Thus, connections between biochemical reactions through the substrate and the product metabolites form complex metabolic networks that may be analyzed using network theory, stoichiometric analysis, and information on protein structure/function and metabolite properties. ${ }^{36}$ In this study, estimates of various enzyme activities in Trp pathways were calculated using the product-to-precursor ratios for each enzyme. The following ratios were used: 5-hydroxytryptophan (5HTP)/Trp for Trp hydroxylase; 5HT/5HTP, 5HT/Trp and tryptamine (Trpa)/Trp for aromatic L-amino acid decarboxylase; $N$-acetylserotonin (NA5HT)/5HTP, NA5HT/5HTP and NA5HT/Trp for serotonin $N$-acetyltransferase; melatonin (Mel)/NA5HT and Mel/5HT for 5-hydroxyindole-O-methyltransferase; 5HIAA/5HT, 5HIAA/5HTP and 5HIAA/Trp for monoamine oxidase and aldehyde dehydrogenase; Kyn/Trp for indoleamine 2,3-dioxygenase and formamidase; and 3OHKy/Kyn and 3OHKy/Trp for kynurenine 3-hydroxylase. The absolute value for each Trp metabolite was used for calculating the ratios. Significant changes in such ratios were based on comparing patients and controls or on treatment with antipsychotics.

\section{Statistical analyses}

All analyte variables were expressed as $\mathrm{ng} \mathrm{ml}^{-1}$ of plasma. Three groups of samples were analyzed: controls (HCs, $n=30$ ), FENNS at BL (FENNS-BL, $n=25$ ) and the same patients after 4 weeks of antipsychotic treatment (FENNS-4w, $n=25$ ). A total of 13 analytes within the Trp pathway were measured and the descriptive statistics were computed for each of the three groups according to the flow chart for data analyses (Figure 2). As not all analytes were normally distributed, hypotheses of no difference between the relative concentrations of each analyte for the $\mathrm{HC}$ and FENNS-BL groups, and for the FENNS-BL and

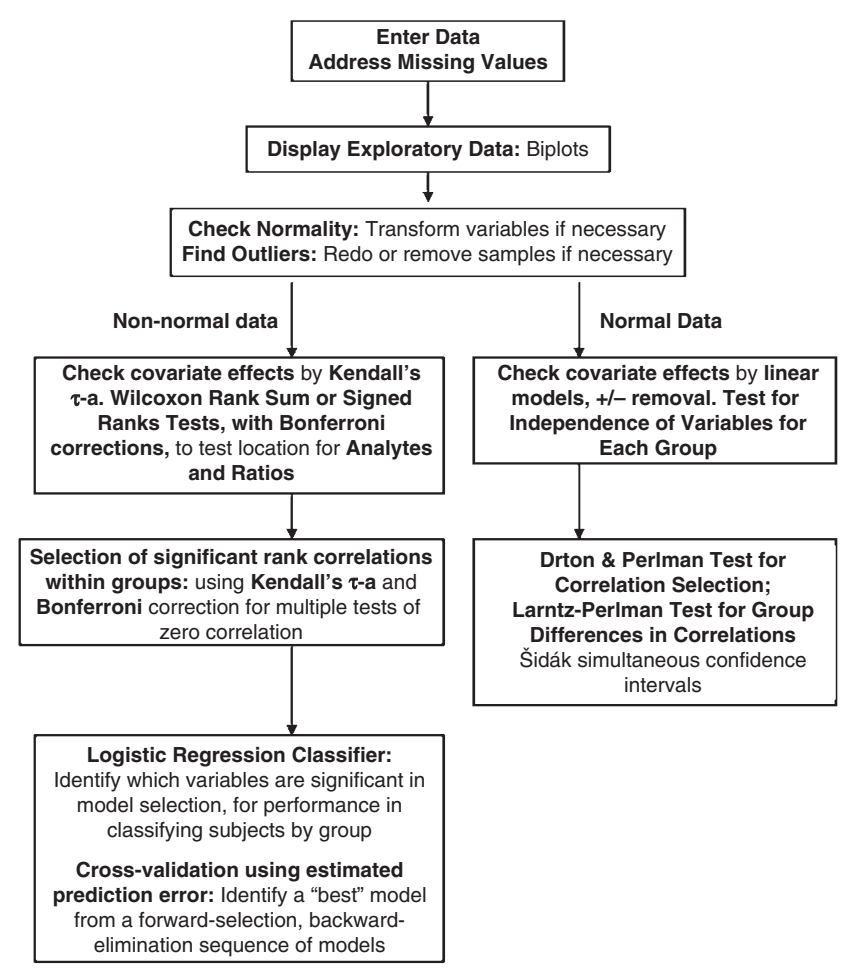

Figure 2 Flow chart of data analyses.

FENNS-4w time points, were tested by nonparametric Wilcoxon rank sum tests and Wilcoxon signed ranks tests, respectively. Some analytes had a number of identical minimum entries that were censored below. When ties were present, the normal approximations for the above Wilcoxon test statistics ${ }^{37}$ were used in the tests. Control of type I error for this multiple testing process was effected by applying the Bonferroni correction.

The raw data were viewed by quantile-quantile normal and $\chi^{2}$ plots, and by variable-pair scatterplots, to assess normality and nonlinear relationships. The majority of the analytes (8 of 13) within the Trp pathway either was, or was able to be transformed to, approximate univariate and multivariate normality by the following small set of strictly increasing (from left to right) functions of the variable (or simply, transformations): natural log (ln), square root (sqrt), quartic root (qurt), negative inverse (inv), negative inverse square root (isq) and negative inverse quartic root (iqu). A correlation test ${ }^{38}$ and Henze and Zirkler's test $^{39}$ were used to assess approximate univariate and multivariate normality, respectively, requiring $P<0.10$ for rejection of normality. Analyte concentration variables with larger numbers of entries censored below could not be transformed to normality and were not entered into this portion of the analysis. The analyte variables with normal distributions were Trp, serotonin, tryptamine, tryptophol, 5HIAA, Mel, kynurenine and 3-OH-kynurenine.

Gender, age and weight, and height or body mass index were available for all but one control. For 
normally distributed data, dependence on covariates within group pairs was checked through linear models. We first examined group differences in weight and height with gender and age as covariates, and then studied group differences in each analyte with each covariate successively included. For the raw, non-normal data, we computed Kendall's ô-a correlations between analytes and the covariates within each group, as a measure of dependence on the covariates. We resolved to remove any dependence reaching significance for $\alpha=0.10$ (two-tailed), subjected to Bonferroni correction for the number of analytes, in subsequent analyses.

All three groupings of data (CTRL, FENNS-BL and FENNS-4w) were tested for complete independence of the Trp pathway variables. ${ }^{40}$ Following Larntz and Perlman ${ }^{41}$ and Drton and Perlman, ${ }^{42}$ we used Fisher's $z$-statistic with Šidák simultaneous 95\% (and 90\%) confidence intervals ${ }^{43}$ to determine which correlations were clearly (or possibly) different from zero. The degrees of freedom were adjusted for multiple correlations rather than for the partial correlations. ${ }^{44}$ These and all other tests on covariance/correlation matrices had their $P$ values checked by Monte Carlo simulation using 10000 randomly generated datasets, because of the asymptotic assumption of these tests and the limited sample sizes in our data. The test statistic and Monte Carlo $P$ values did not differ greatly, but the Monte Carlo $P$ values were taken as more accurate.

We also used the rank correlation method of Kendall to examine associations among all 13 untransformed analyte variables, within each volunteer group. This method tests the null hypothesis of no rank correlation between the two variables whose distribution is not known, and can accommodate tied or censored values up to the extreme case of variables with dichotomous values. ${ }^{45}$ We tested the $13^{*}(13-1) /$ $2=78$ Kendall $\tau$ correlations against the two-sided alternative, using Bonferroni-corrected $\alpha=0.05$ (or $\alpha=0.10$ ), to determine which correlations were clearly (or possibly) different from zero, for each group separately.

Finally, the ability of the analytes to classify volunteers by group membership was examined by logistic regression. ${ }^{46}$ A sequence of models was generated by forward selection/backward elimination. Models were assessed by deviance reduction, as well as by 5 - and 10-fold cross-validation, with prediction error estimated by the negative log-likelihood loss function. ${ }^{47} \mathrm{~K}$-fold cross-validation is used to avoid choosing models suggested by random aspects of the data. One divides the $\mathrm{N}$ data samples randomly into $\mathrm{K}$ nearly equal portions, and leaves each portion out, successively, while making $\mathrm{K}$ estimations of the model parameters, giving $\mathrm{K}$ models. Then each of the $\mathrm{K}$ models is used to compute the estimated prediction error (EPE) for the corresponding left-out portion of the data. The means and standard errors of the K EPE values are then plotted as the process is repeated for the original large sequence of models (with different numbers of analyte variables). A common procedure for identifying a 'best' model is to first find the model with the lowest EPE value (see Figure 2), and then to select the smallest model with EPE within one standard error of the lowest EPE value. ${ }^{47}$

\section{Results}

Tests of difference in the group locations

Descriptive statistics for all the analytes, by group, appear in Table 2. Note that 5HTP, NA5HT, NM5HT, 3OHKy, anthranillic acid (ANA) and 3-hydroxyanthranillic acid (3OHANA) had medians much lower than their means, and sometimes identical to first quartile values. Substantial numbers of samples for these analytes had values measured at the same (analyte-specific) lower threshold, that is, censored below. ANA presented the extreme case with almost all samples censored below 11.15.

Testing demographic covariates. Of the covariates, height, weight and age were normally distributed, but body mass index was not. There were significant differences $(P<0.05)$ between the HC and FENNS groups in both height and weight even after the (significant) covariate 'gender' was entered, but age had no significant contribution (see Table 1). For the raw, non-normal data, we found no significant $(\alpha=0.10)$ Kendall's $\tau$ correlations between analytes and the covariates (gender, age and body mass index) within groups or within group pairs after Bonferroni correction for the number of analytes. Therefore, we did not correct for any effects of covariates in the nonparametric analyses to follow.

Non-parametric location testing. The Wilcoxon ranksum tests showed higher $N$-acetylserotonin in FENNSBL compared with HC $(P=0.0077$, a 'trend-level' difference after Bonferroni correction of an $\alpha$ of 0.05 to obtain 0.0038 , or 0.10 to obtain 0.0077 , Table 2). After antipsychotic drug treatment, the mean level of $\mathrm{N}$-acetylserotonin was reduced, although the difference was not statistically significant (Wilcoxon signed rank tests). Similarly, the difference between FENNS$4 \mathrm{w}$ and HC groups was also not statistically significant $(P=0.4570)$. However, the levels of both Mel $(P=0.0034)$ and $\operatorname{Trp}(P=0.0070)$ were reduced and possibly reduced, respectively, in FENNS-4w compared with HC, but not to the FENNS-BL patients (Table 2).

On the other hand, these groups did differ in post-hoc comparisons involving a set of ratios of analytes (Table 3), selected as described above. The ratios of NA5HT/Trp and $\mathrm{Mel} / 5 \mathrm{HT}$ were significantly higher ( $P$-values $<0.0029)$ and the ratio of Mel/NA5HT was significantly $(P=0.0011)$ lower in FENNS-BL than in HC volunteers (Table 3). After antipsychotic drug treatment, however, such differences were no longer present.

Logistic regression group classifier. Furthermore, we built a classification model using logistic regression 
Table 2 Descriptive statistics of metabolites in tryptophan pathway

\begin{tabular}{|c|c|c|c|c|c|c|}
\hline Groups & Mean & Median & Firstqrt & Thirdqrt & $\mathrm{P}$-value (HC vs FENNS) & P-value (BL vs $4 w$ ) \\
\hline \multicolumn{7}{|l|}{ 1. $H C$} \\
\hline Trp & $7627.62^{\mathrm{a}}$ & 7724.11 & 5178.10 & 9960.99 & & \\
\hline $5 \mathrm{HTP}$ & 2.41 & 1.08 & 0.73 & 3.49 & & \\
\hline $5 \mathrm{HT}$ & 199.71 & 144.68 & 101.96 & 268.85 & & \\
\hline NA5HT & 1.17 & 0.78 & 0.78 & 1.64 & & \\
\hline NM5HT & 0.18 & 0.01 & 0.01 & 0.22 & & \\
\hline 5HIAA & 18.05 & 16.81 & 11.30 & 22.55 & & \\
\hline Mel & 1.03 & 0.88 & 0.69 & 1.28 & & \\
\hline Kyn & 160.99 & 137.96 & 108.79 & 235.27 & & \\
\hline 3OHKy & 10.00 & 8.46 & 5.17 & 13.46 & & \\
\hline ANA & 3.75 & 3.58 & 3.58 & 3.58 & & \\
\hline 3OHANA & 1.02 & 0.26 & 0.26 & 1.15 & & \\
\hline Trpa & 18.75 & 17.41 & 13.67 & 23.59 & & \\
\hline Tpol & 6.33 & 5.05 & 3.48 & 8.47 & & \\
\hline \multicolumn{7}{|c|}{ 2. FENNS-BL } \\
\hline $\operatorname{Trp}$ & 6426.00 & 6479.71 & 3525.37 & 8243.70 & 0.0692 & \\
\hline 5НTP & 4.59 & 0.73 & 0.73 & 0.95 & 0.0950 & \\
\hline $5 \mathrm{HT}$ & 237.75 & 221.84 & 129.91 & 318.84 & 0.3012 & \\
\hline NA5HT & 2.43 & 1.73 & 0.78 & 3.04 & $0.0077^{b}$ & \\
\hline NM5HT & 0.68 & 0.01 & 0.01 & 0.36 & 0.7271 & \\
\hline 5HIAA & 19.21 & 13.48 & 10.45 & 22.35 & 0.4967 & \\
\hline Mel & 0.80 & 0.73 & 0.54 & 1.13 & 0.0732 & \\
\hline Kyn & 125.33 & 95.95 & 69.47 & 156.71 & 0.0772 & \\
\hline 3ОНКу & 8.17 & 4.65 & 2.85 & 11.32 & 0.1762 & \\
\hline ANA & 3.84 & 3.58 & 3.58 & 3.58 & 0.8963 & \\
\hline 3OHANA & 1.08 & 0.56 & 0.26 & 1.51 & 0.1536 & \\
\hline Trpa & 17.21 & 14.29 & 12.51 & 16.97 & 0.1447 & \\
\hline Tpol & 5.41 & 4.37 & 3.70 & 5.47 & 0.5074 & \\
\hline \multicolumn{7}{|c|}{ 3. FENNS-4W } \\
\hline $\operatorname{Trp}$ & 5469.01 & 4498.95 & 3350.59 & 7604.97 & 0.0070 & 0.1336 \\
\hline 5НТР & 1.37 & 0.73 & 0.73 & 1.50 & 0.0513 & 0.8465 \\
\hline $5 \mathrm{HT}$ & 182.22 & 146.35 & 93.35 & 249.26 & 0.5627 & 0.0203 \\
\hline NA5HT & 1.52 & 1.02 & 0.78 & 2.06 & 0.4570 & 0.2054 \\
\hline NM5HT & 0.65 & 0.01 & 0.01 & 0.81 & 0.5337 & 0.5917 \\
\hline 5HIAA & 16.98 & 10.80 & 7.20 & 13.89 & 0.0144 & 0.2635 \\
\hline Mel & 0.70 & 0.67 & 0.42 & 0.87 & $0.0034^{b}$ & 0.1563 \\
\hline Kyn & 110.64 & 90.74 & 60.56 & 137.42 & 0.0137 & 0.2872 \\
\hline 3ӦНу & 7.73 & 3.98 & 1.24 & 11.19 & 0.0718 & 0.7510 \\
\hline ANA & 4.05 & 3.58 & 3.58 & 3.58 & 0.8963 & 1.0000 \\
\hline $3 \mathrm{OHAN}$ & 1.14 & 0.90 & 0.26 & 2.15 & 0.0689 & 0.8916 \\
\hline Trpa & 16.32 & 15.12 & 12.59 & 18.91 & 0.1750 & 0.7915 \\
\hline Tpol & 5.85 & 5.08 & 4.14 & 7.42 & 0.8210 & 0.0755 \\
\hline
\end{tabular}

Abbreviations: ANA, anthranillic acid; BL, baseline; FENNS, first-episode neuroleptic-naive patients with schizophrenia; HC, healthy control; 5-HIAA, 5-hydroxyindoleacetic acid; 5-HT, serotonin; 5HTP, 5-hydroxytryptophan; Kyn, kynurenine; Mel, melatonin; NA5HT, $N$-acetyl serotonin; NM5HT, $N$-methylserotonin; 3-OHAN, 3-hydroxyanthranillic acid; 3OHKy, 3hydroxykynurenine; Tpol; tryptophol; Trp, tryptophan; Trpa, tryptamine; 4w, 4-week treatment with antipsychotic drugs. ${ }^{\mathrm{a}}$ Each value represents $\mathrm{ng} \mathrm{m \textrm {m } ^ { - 1 }}$.

bignificant difference after Bonferroni correction of an $\alpha$ of 0.05-0.0038, or 0.10-0.0077.

to assess the performance of the Trp pathway analytes in separating the groups in space. In comparing HC with FENNS-BL, higher NA5HT (deviance reduction 10.16 on $1 \mathrm{df}, \operatorname{Pr}\left(\chi^{2}\right)=0.001$ ), and higher NM5HT (additional deviance reduction 4.72 on $1 \mathrm{df}, \operatorname{Pr}\left(\chi^{2}\right)=$ 0.03) indicated membership in the FENNS-BL group over the HC group. The NA5HT and NM5HT model correctly classified 17 of 25 (68\%) FENNS-BL and 24 of $30(80 \%)$ HC. However, both 5- and 10-fold crossvalidation found that the prediction error estimates (here, the negative log-likelihood loss function) of models with more than one variable were not any better than that of the single-variable NA5HT model (Figure 3). Note that this result is in agreement with the significant finding for NA5HT by the Wilcoxon rank-sum test (Table 2). In comparing $\mathrm{HC}$ with 
Table 3 Comparisons of ratios of product to precursor in tryptophan pathway between healthy controls and FENNS patients and between FENNS patients before and after antipsychotic treatment

\begin{tabular}{|c|c|c|c|c|c|c|}
\hline \multirow[t]{2}{*}{ Ratios } & \multirow[t]{2}{*}{$\mathrm{HC}$} & \multirow[t]{2}{*}{ FENNS-BL } & \multirow[t]{2}{*}{ FENNS-4W } & \multicolumn{3}{|c|}{$\mathrm{P}$-value } \\
\hline & & & & $H C$ vs $B L^{\mathrm{a}}$ & $H C$ vs $4 w^{\mathrm{a}}$ & $B L$ vs $4 w^{\mathrm{b}}$ \\
\hline 5HTP/Trp & $(3.00 \pm 2.43) \mathrm{E}-04$ & $(7.49 \pm 19.0) \mathrm{E}-04$ & $(3.91 \pm 8.23) \mathrm{E}-04$ & 0.2355 & 0.5972 & 0.2996 \\
\hline 5HT/Trp & $0.03 \pm 0.01^{\mathrm{c}}$ & $0.04 \pm 0.04$ & $0.04 \pm 0.04$ & 0.0074 & 0.2224 & 0.2200 \\
\hline 5HT/5HTP & $147 \pm 146$ & $244 \pm 191$ & $183 \pm 180$ & 0.0331 & 0.4449 & 0.0422 \\
\hline NA5HT/Trp & $(2.00 \pm 1.47) \mathrm{E}-04$ & $(4.44 \pm 3.68) \mathrm{E}-04$ & $(3.54 \pm 2.77) \mathrm{E}-04$ & $0.0025^{\mathrm{d}}$ & 0.0144 & 0.4742 \\
\hline NA5HT/5HTP & $1.09 \pm 1.01$ & $2.59 \pm 2.30$ & $1.75 \pm 1.69$ & 0.0083 & 0.0700 & 0.2012 \\
\hline NA5HT/5HT & $0.01 \pm 0.02$ & $0.02 \pm 0.04$ & $0.02 \pm 0.04$ & 0.1977 & 0.3091 & 0.7510 \\
\hline $\mathrm{Mel} / \mathrm{Trp}$ & $(1.40 \pm 0.04) \mathrm{E}-04$ & $(1.30 \pm 0.03) \mathrm{E}-04$ & $(1.34 \pm 0.04) \mathrm{E}-04$ & 0.3963 & 0.3870 & 0.7510 \\
\hline $\mathrm{Mel} / 5 \mathrm{HTP}$ & $0.74 \pm 0.47$ & $0.89 \pm 0.58$ & $0.77 \pm 0.50$ & 0.3255 & 0.9265 & 0.3254 \\
\hline $\mathrm{Mel} / 5 \mathrm{HT}$ & $0.01 \pm 0.01$ & $0.02 \pm 0.07$ & $0.01 \pm 0.03$ & $0.0027^{d}$ & 0.1311 & 0.1311 \\
\hline Mel/NA5HT & $1.07 \pm 0.64$ & $0.57 \pm 0.47$ & $0.65 \pm 0.47$ & $0.0011^{\mathrm{d}}$ & 0.0045 & 0.5782 \\
\hline 5HIAA/Trp & $(2.35 \pm 0.91) \mathrm{E}-03$ & $(3.77 \pm 4.34) \mathrm{E}-03$ & $(3.82 \pm 5.28) \mathrm{E}-03$ & 0.1185 & 0.5074 & 0.4578 \\
\hline 5HIAA/5HTP & $12.39 \pm 8.05$ & $21.69 \pm 22.07$ & $17.53 \pm 23.22$ & 0.1185 & 0.7692 & 0.3666 \\
\hline 5HIAA/5HT & $0.17 \pm 0.32$ & $0.15 \pm 0.27$ & $0.21 \pm 0.33$ & 0.2223 & 0.7060 & 0.2200 \\
\hline Kyn/Trp & $0.02 \pm 0.01$ & $0.02 \pm 0.01$ & $0.02 \pm 0.01$ & 0.3424 & 0.6326 & 0.8325 \\
\hline 3OHKy/Trp & $(1.23 \pm 0.68) \mathrm{E}-04$ & $(1.10 \pm 0.69) \mathrm{E}-03$ & $(1.29 \pm 1.29) \mathrm{E}-04$ & 0.4967 & 0.2098 & 0.7510 \\
\hline $3 \mathrm{OHKy} / \mathrm{Kyn}$ & $0.06 \pm 0.03$ & $0.05 \pm 0.03$ & $0.06 \pm 0.04$ & 0.6811 & 0.3778 & 0.8532 \\
\hline Trpa/Trp & $(2.73 \pm 1.27) \mathrm{E}-03$ & $(2.99 \pm 1.20) \mathrm{E}-03$ & $(3.39 \pm 1.13) \mathrm{E}-04$ & 0.3963 & 0.0070 & 0.1409 \\
\hline
\end{tabular}

Abbreviations: BL, baseline; FENNS, first-episode neuroleptic-naive patients with schizophrenia; 5HIAA, 5-hydroxyindoleacetic acid; 5-HT, serotonin; 5HTP, 5-hydroxy tryptophan; Kyn, kynurenine; Mel, melatonin; NA5HT, $N$-acetyl serotonin; 3OHKy, 3-hydroxykynurenine; Trp, tryptophan; Trpa, tryptamine; 4w, 4-week treatment with antipsychotic drugs.

${ }^{a}$ Wilcoxon rank sum test, two-sided.

${ }^{\mathrm{b}}$ Wilcoxon signed rank sum test; two-sided.

${ }^{\mathrm{c}}$ Mean and standard deviation.

${ }^{\mathrm{d}}$ Significance with $P<0.0029$ after the Bonferroni correction.

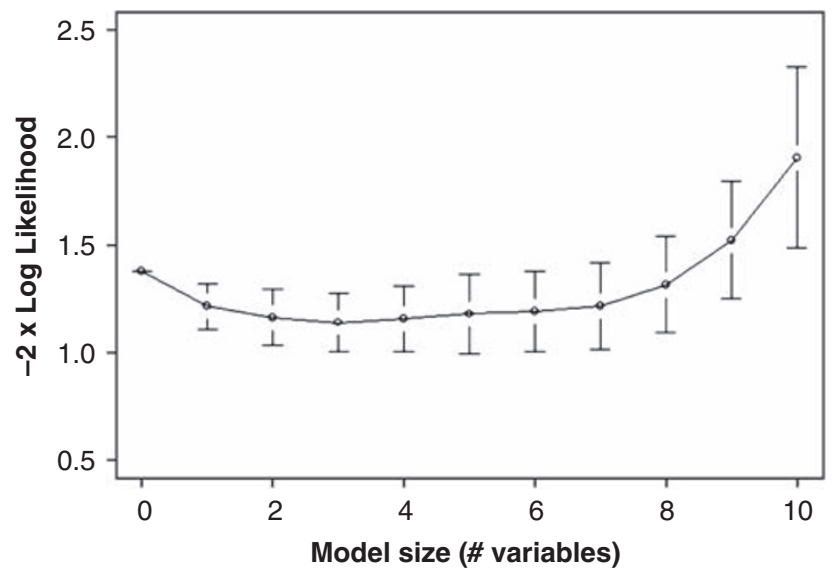

Figure 3 Cross-validation (fivefold) for the logistic regression classifier of HC vs FENNS-BL groups. A series of models was chosen by forward selection/backward elimination using as criterion the minimizing of the estimated prediction error $(-2 \times \log$ likelihood), displayed for each model \pm its own standard error. Note that the 3 -variable model has the lowest value, but that a practical selection rule (see text) favors choosing the 1-variable model (NA5HT): essentially as good and more parsimonious. HC, healthy controls; FENNS-BL, first-episode neuroleptic-naive schizophrenic patients at baseline; NA5HT, $N$-acetyl serotonin.
FENNS-4w, lower Mel levels (deviance reduction 9.37 on $1 \mathrm{df}, \operatorname{Pr}\left(\chi^{2}\right)=0.002$ ) and higher NM5HT (deviance reduction 5.92 on $1 \mathrm{df}, \operatorname{Pr}\left(\chi^{2}\right)=0.01$ ) indicated membership in the FENNS-4w group as opposed to the HC group. The model correctly classifies 17 of 25 (68\%) FENNS-4w patients and 21 of $30(70 \%)$ HC volunteers, therefore neither model achieved highly accurate classification. The cross-validation results found the Mel and NM5HT model lowest in EPE, but no better than the one-variable, 'best' model with Mel only. The graph (not shown) is essentially the same as in Figure 3. None of the analytes made a significant contribution to a model attempting to classify the drug-naive state vs drug-treated state of the patients, whether assessed by deviance reduction or by cross-validation.

\section{Testing for non-zero correlations}

For the eight normally distributed (transformed) analyte variables, we tested the dependence of analytes on covariates and the 'group' variable within group pairs. We found no differences that were significant after Bonferroni correction for the number of analytes $(P<0.10 / 8=0.0125)$, similar to the case with the raw, non-normal data above. 
Table 4 Within group correlations by the Drton-Perlman procedure and by the Kendall $\tau$ method

\begin{tabular}{|c|c|c|c|c|c|c|c|c|c|c|c|c|c|}
\hline \multirow{2}{*}{\multicolumn{2}{|c|}{ Metabolites $^{\mathrm{a}}$}} & \multicolumn{6}{|c|}{ Drton-Perlman correlations ${ }^{b}$} & \multicolumn{6}{|c|}{ Kendall's $\tau$ rank correlations ${ }^{\mathrm{b}}$} \\
\hline & & \multicolumn{2}{|c|}{$H C$} & \multicolumn{2}{|c|}{ FENNS-BL } & \multicolumn{2}{|c|}{ FENNS-4W } & \multicolumn{2}{|c|}{$H C$} & \multicolumn{2}{|c|}{$F E N N S-B L$} & \multicolumn{2}{|c|}{ FENNS-4w } \\
\hline$I$ & II & $\mathrm{r}$ & P-value & $\mathrm{r}$ & P-value & $\mathrm{r}$ & $\mathrm{P}$-value $\mathrm{c}$ & $\tau$ & P-value ${ }^{\mathrm{d}}$ & $\tau$ & P-value $\mathrm{d}^{\mathrm{d}}$ & $\tau$ & P-value \\
\hline \multicolumn{14}{|c|}{ Significant correlations were obtained among all three groups } \\
\hline Trp & Mel & 0.8282 & $<0.0001$ & 0.8193 & $<0.0001$ & 0.8008 & 0.0001 & 0.6138 & 0.0002 & 0.7245 & $<0.0001$ & 0.6600 & 0.0003 \\
\hline Trp & Kyn & 0.8656 & $<0.0001$ & 0.8434 & $<0.0001$ & 0.7976 & 0.0001 & 0.7103 & $<0.0001$ & 0.6912 & 0.0001 & 0.7133 & 0.0001 \\
\hline Trp & 3-ОНКу & 0.7615 & $<0.0001$ & 0.7702 & 0.0001 & 0.7178 & 0.0012 & 0.5356 & 0.0027 & 0.6420 & 0.0007 & 0.5710 & 0.0056 \\
\hline Trp & Trpa & 0.5283 & 0.0722 & 0.7353 & 0.0008 & 0.6541 & 0.0113 & 0.4575 & 0.0316 & 0.5910 & 0.0031 & 0.5733 & 0.0051 \\
\hline \multicolumn{14}{|c|}{ Significant correlations were obtained only in HC, but not in FENNS groups } \\
\hline $5 \mathrm{HT}$ & $\operatorname{Trp}$ & 0.6387 & 0.0044 & 0.2355 & 0.9998 & 0.2461 & 0.9995 & 0.5126 & 0.0058 & 0.2104 & 1 & 0.1000 & 1 \\
\hline $5 \mathrm{HT}$ & Mel & 0.6336 & 0.0051 & 0.1028 & 1 & 0.0006 & 1 & 0.4667 & 0.0242 & 0.1600 & 1 & -0.0400 & 1 \\
\hline $5 \mathrm{HT}$ & Kyn & 0.5859 & 0.0200 & 0.2407 & 0.9996 & 0.0305 & 1 & 0.4529 & 0.0361 & 0.1800 & 1 & 0.0133 & 1 \\
\hline $5 \mathrm{HT}$ & Trpa & 0.5662 & 0.0314 & 0.0991 & 1 & -0.0114 & 1 & 0.4115 & 0.1101 & 0.1600 & 1 & 0.0600 & 1 \\
\hline 5-HIAA & Trp & 0.7820 & $<0.0001$ & 0.1429 & 1 & 0.3108 & 0.9798 & 0.6874 & $<0.0001$ & 0.0902 & 1 & 0.3800 & 0.4785 \\
\hline 5-HIAA & Mel & 0.6474 & 0.0032 & 0.2473 & 0.9994 & 0.1450 & 1 & 0.5310 & 0.0032 & 0.1667 & 1 & 0.2400 & 0.9997 \\
\hline 5-HIAA & Kyn & 0.7068 & 0.0004 & 0.2681 & 0.9977 & 0.3413 & 0.9387 & 0.5540 & 0.0014 & 0.1333 & 1 & 0.3067 & 0.9302 \\
\hline 5-HIAA & 3-OHKy & 0.6980 & 0.0006 & 0.2654 & 0.9980 & 0.4107 & 0.6940 & 0.4713 & 0.0211 & 0.1342 & 1 & 0.3573 & 0.6474 \\
\hline
\end{tabular}

Abbreviations: BL, baseline; FENNS, first-episode neuroleptic-naive patients with schizophrenia; HC, healthy control; 5-HIAA, 5-hydroxyindoleacetic acid; 5-HT, serotonin; Kyn, kynurenine; Mel, melatonin; 3OHKy, 3-hydroxykynurenine; Tpol; tryptophol; Trp, tryptophan; Trpa, tryptamine; 4w, 4-week treatment with antipsychotic drugs.

an case of Drton-Perlman procedure, sqrtTrp, sqrt5HT, iqu5HIAA, iquMel, ln3-OHKy, qurtKyn and iquTrpa were used for correlations.

${ }^{\mathrm{b}}$ Tests for zero correlation, two-sided. See text for metabolite transformations.

'Šidák simultaneous $P$-values, significance at $P<0.05$.

${ }^{\mathrm{d}} P$ times Bonferroni correction factor, for comparison, significance at $P<0.05$.

As the tests for total independence were highly significant for all three groups (Schott's test; HC: $z=35.18, P=0$; FENNS-BL: $z=19.78, P=0$; FENNS$4 \mathrm{w}: z=16.62, P=0$ ), we sought to determine which multivariate correlation coefficients were significantly different from zero. As suggested by Drton and Perlman, ${ }^{42}$ we chose to label correlations with $P<0.05$ as significant, and to regard a group of correlations for which $0.05<P<0.10$, as having indeterminate significance.

Within the Trp pathway, all three groups had highly significant correlations between Trp and its metabolites, Mel, kynurenine, 3-hydroxykynurenine and tryptamine (Table 4). By contrast, significant correlations between serotonin and Trp, Mel, kynurenine or tryptamine were only shown in normal controls, and not in FENNS patients at BL or in FENNS patients after 4-week treatment with antipsychotic drugs (Figure 4). Similarly, in the HC group 5HIAA was highly correlated with Trp, Mel, kynurenine or 3-hydroxykynurenine; these correlations were not found to be significant for either patient group (Figure 5). For easier comparison, Šidák simultaneous (corrected) $P$ values rather than confidence intervals are presented in Table 4.

As the original data were not all normally distributed or transformable to approximate normality, we also computed the non-parametric Kendall's $\tau$ rank correlations, with Bonferroni correction for the control of type I error. The parametric results were largely reproduced (Table 4), with the only slight differences noted for the 5HT-Trpa correlation, which was significant for the HC group using the Drton-Perlman procedure but not using the Kendall $\tau$ method.

In addition, the Larntz-Perlman method was used to produce simultaneous Šidák 95\% confidence intervals to test explicitly for differences in the non-null multiple correlations between two groups. These correlation confidence intervals exclude 0, and produce a simultaneous Šidák $P$-value of 0.036 , for $\alpha=0.05$, only for the correlation between the transformed Trp and 5HIAA variables, when compared between the HC and FENNS-BL groups (Figure 5).

\section{Discussion}

Lacking control mechanism in the biosynthesis and catabolism of serotonin

Tryptophan degradation constitutes primarily three branches including serotonin, kynurenine and tryptamine pathways (Figure 6). When compared with serotonin or 5HIAA levels (Table 4), Trp (precursor) and Mel (metabolite) levels indicate that the metabolic control of relative metabolite ratios is occurring in $\mathrm{HC}$ volunteers, but these controls are completely lost for either FENNS patient groups. Although levels of serotonin (or 5HIAA) were also significantly 

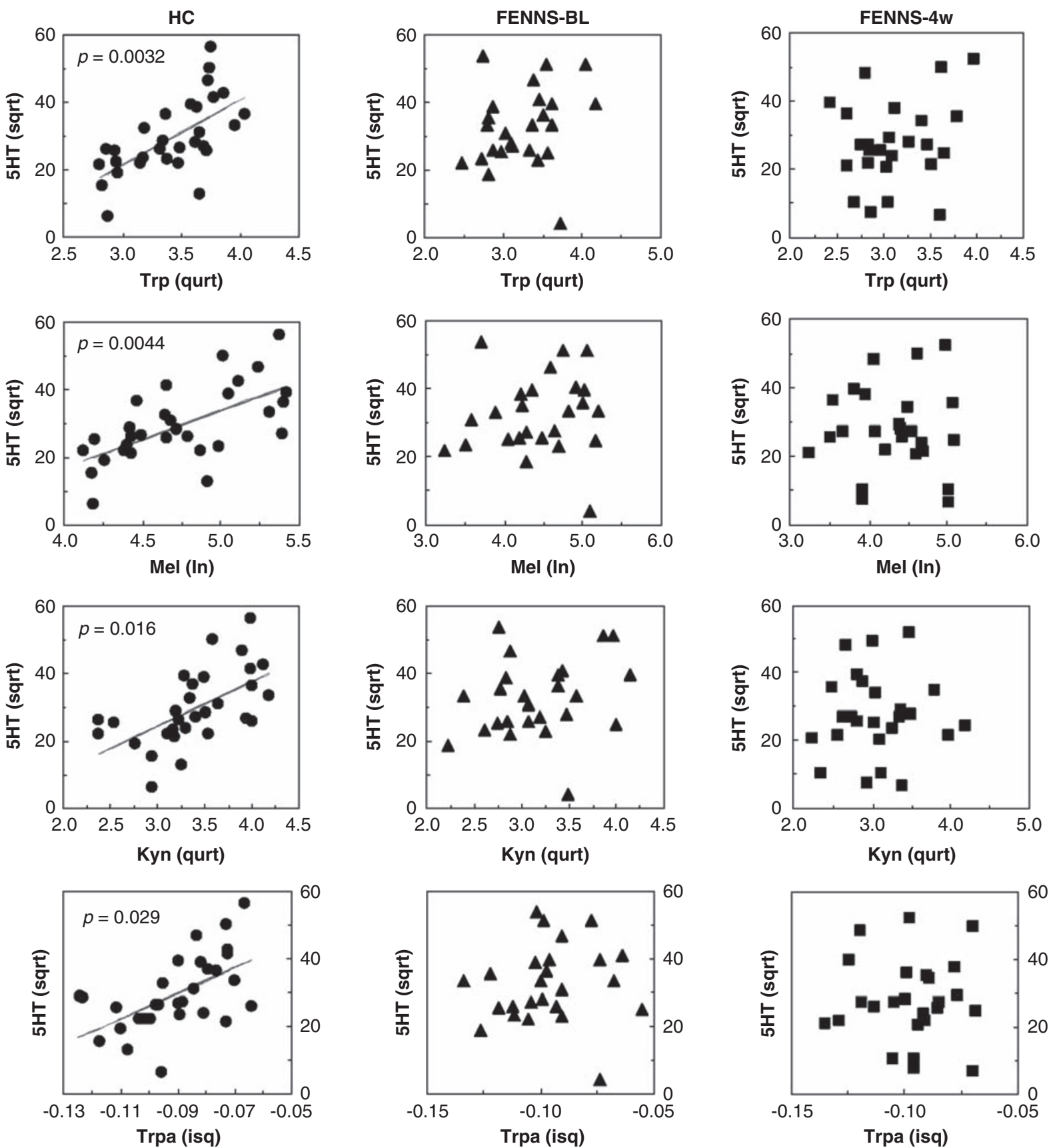

Figure 4 Significant correlations of serotonin (5HT) to tryptophan (Trp), melatonin (Mel), Kynurenine (Kyn) or tryptamine (Trpa) in normal controls, but not in FENNS patients at baseline (BL) and FENNS patients after 4-week (4w) treatment with antipsychotic drugs. The $P$ values were calculated by Monte Carlo simulated probability of the statistic (good approximation for all sample sizes). FENNS, first-episode neuroleptic-naive schizophrenic; sqrt, square root; qurt, quartic root; ln, natural log; isq, negative inverse square root.

correlated with kynurenine, 3-hydroxykynurenine or tryptamine, such correlations are likely secondary to the significant correlations between Trp and the above metabolites (Table 4). Earlier, Payne et al. ${ }^{48}$ have shown that non-schizophrenics had significant positive correlations between dietary Trp and kynurenic acid or xanthurenic acid, and between niacin ingestion and kynurenic acid, 5HIAA or xanthurenic acid, whereas the schizophrenics did not. Taken together, a control mechanism used by HC volunteers seems to be impaired in FENNS patients to regulate Trp metabolites through the serotonin pathway.

\section{Upregulation of $\mathrm{N}$-acetylserotonin synthesis from serotonin}

As increased levels of NA5HT and the ratio of NA5HT to its precursor (Trp), as well as decreased ratio of Mel (metabolite) to NA5HT were also shown in FENNS-BL compared with HC volunteers, it is likely that 

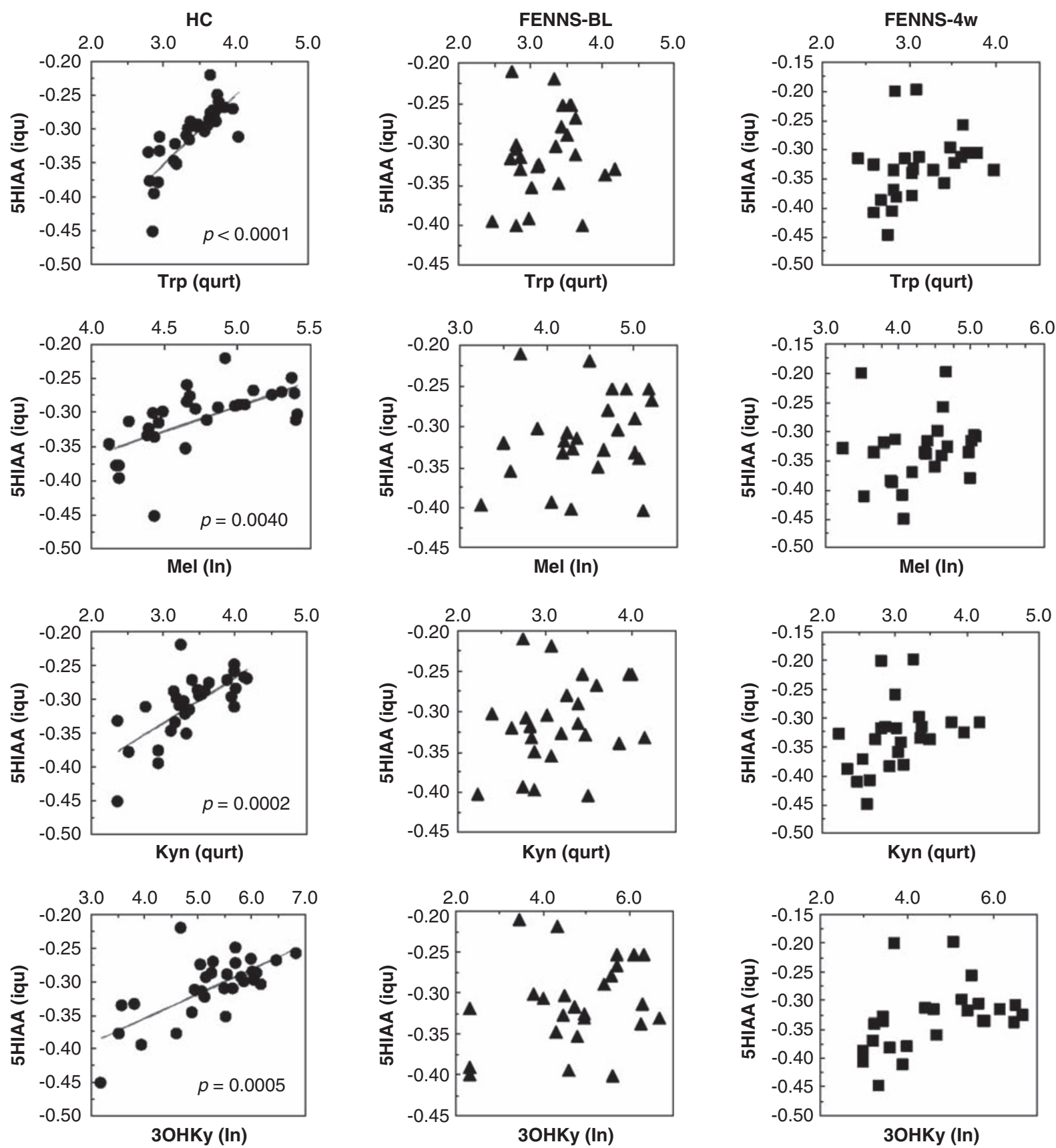

Figure 5 Significant correlations of 5-hydroxyindoleacetic acid (5HIAA) to tryptophan (Trp), melatonin (Mel), Kynurenine (Kyn) or 3-hydroxy-kynurenine (3OHKy) in normal control subjects, but not in FENNS patients at baseline and FENNS patients after 4-week treatment with antipsychotic drugs. The $P$ values were calculated by Monte Carlo simulated probability of the statistic (good approximation for all sample sizes). FENNS, first-episode neuroleptic-naive schizophrenic; iqu, negative inverse quartic root; qurt, quartic root; ln, natural log.

conversion of serotonin to NA5HT (Figure 6) is specifically upregulated in the FENNS-BL patients.

In the pineal gland, outside the blood-brain barrier, synthesis of serotonin and conversion to NA5HT and Mel occur. ${ }^{49}$ This pathway is ordinarily upregulated by sympathetic activity with origin through suprachiasmatic nucleus, hypothalamus and superior cervical ganglion, in the absence of light. The $\alpha$ - and $\beta$-noradrenergic activation of the rate-limiting enzyme, arylalkylamine $N$-acetyltransferase, followed by the rapid transfer of a methyl group to $\mathrm{N}$-acetylserotonin through hydroxyindol-O-methyltransferase, generates far higher levels of Mel at night than during daytime. ${ }^{50}$ To avoid the effect of diurnal rhythm in the Mel level, all blood samples were collected in the morning after overnight fasting.

Although arylalkylamine $N$-acetyltransferase and hydroxyindol-O-methyltransferase have been localized primarily to the pineal and retinal tissues, these enzymes and Mel receptors are also found in other 


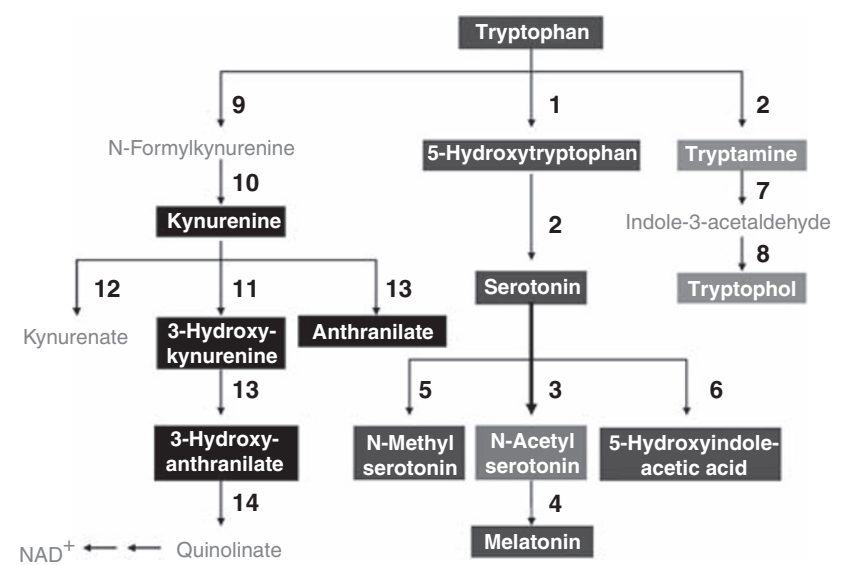

Figure 6 Tryptophan metabolic pathways. The bold arrow indicates that pathway may be upregulated in first-episode neuroleptic-naive patients with schizophrenia. (1) Tryptophan hydroxylase; (2) Aromatic L-amino acid decarboxylase; (3) Serotonin $N$-acetyltransferase; (4), 5-Hydroxyindole- $O$-methyltransferase; (5) Serotonin $N$-methyltransferase; (6) monoamine oxidase and aldehyde dehydrogenase; (7) Monoamine oxidase; (8) Alcohol dehydrogenase; (9) Tryptophan 2,3-dioxygenase; (10) Formamidase; (11) Kynurenine 3-hydroxylase; (12) Kynurenine transaminase; (13) Kynureninase; (14) 3-Hydroxyanthranilate oxygenase. Also see footnote of Table 2.

locations including brain and peripheral tissues. ${ }^{50-52}$ Interestingly, Mel concentration is 20 times higher in the third ventricle CSF than in plasma. ${ }^{53}$ Tricoire et al. ${ }^{54}$ further showed that Mel is released into the pineal recess (a third ventricle evagination penetrating into the pineal gland) and is then distributed throughout the ventricular system to possibly reach the periventricular binding sites. In addition to the regulation of the circadian rhythms, Mel has been linked to many putative effects resulting from a central action, ${ }^{55}$ for example, enhancement of sleep and immune function, neuroprotection during nerve degeneration and seasonal control of luteinizing hormone secretion.

The possibly elevated NA5HT may in part result from excessive sympathetic tone in the acutely psychotic patients. It is possible that transfer of NA5HT and Mel from the brain compartment and other sites to the peripheral circulation could also have contributed to the findings.

\section{Oxidative stress and neuroprotection}

Schizophrenia is a remarkably complex disorder with a multitude of behavioral and biological perturbations. A multiplicity of theories have been proposed over the years that aim to conceptualize the pathological processes inherent to schizophrenia. ${ }^{2}$ Whether these diverse alterations are independent biological processes or a result of a more fundamental pathology has yet to be determined.

Biological systems have evolved complex protective strategies against free radical toxicity. Under physiological conditions the potential for free radicalmediated damage is kept in check by the antioxidant defense system, comprising a series of enzymatic and non-enzymatic components. These enzymes act cooperatively at different sites in the free radical pathways. Oxidative stress in the brain occurs when the generation of reactive oxygen species overrides the ability of endogenous antioxidant systems to remove excess reactive oxygen species subsequently leading to cellular damage. ${ }^{56}$ Increasing evidence suggests that mitochondrial pathology and oxidative stress may be the most critical component in the pathophysiology and outcome of schizophrenia. ${ }^{57-61}$ Recently, $\mathrm{we}^{62}$ have further observed that a dynamic state is kept in check during redox coupling under normal conditions. By contrast, lack of such correlations in brains with schizophrenia point to a disturbance of redox coupling mechanisms in the antioxidant defense system, possibly resulting from a decreased level of glutathione (GSH) and age-related decreases of oxidized GSH and glutathione reductase activities. Taken together, our earlier data showing altered membrane dynamics and antioxidant defense system enzyme activities, and findings of abnormal GSH gene ${ }^{63}$ are consistent with the notion of free radical-mediated neurotoxicity in schizophrenia.

Both $N$-acetylserotonin and $\mathrm{Mel}$ are indole compounds, which are known to act as a scavenger of reactive oxygen species such as hydroxyl radical, hydrogen peroxide, alkoxyl radical, hypochlorous acid or singlet oxygen. ${ }^{64-66}$ Moreover, Mel may upregulate the endogenous antioxidant defense system by stimulating glutathione peroxidase ${ }^{67,68}$ and superoxide dismutase. ${ }^{69}$ In addition, the ability to inhibit lipid peroxidation by both compounds can protect long-chain polyunsaturated fatty acids in biological membranes. ${ }^{70-72}$ In contrast, other studies indicated that Mel may be a relatively inactive antioxidant. ${ }^{73-76}$ On the other hand, $N$-acetylserotonin is a better extra- and intra-cellular antioxidant than Mel. ${ }^{74,76,77}$ Considering $\mathrm{N}$-acetylserotonin as a potent antioxidant, such increases in $\mathrm{N}$-acetylserotonin might be a compensatory response to increased oxidative stress in schizophrenia.

\section{Effect of antipsychotic treatment on Trp depletion} Using Wilcoxon signed rank tests and the LarntzPerlman procedure, respectively, we found no significant differences in either levels or correlations of 13 Trp metabolites between FENNS-BL and FENNS-4w patients. However, there was a significant reduction of Mel and a 'trend level' decrease of Trp in FENNS4w compared with HC volunteers (Table 2). As Trp is an essential amino acid, a slightly lower dietary intake in the FENNS group may have occurred. The effects of abnormally high levels of sympathetic arousal during acute psychosis can diminish markedly with antipsychotic treatment after 4 weeks, the time at which our samples showed lower Mel levels. Still, paired tests of Mel and NA5HT in FENNS patients did not show changes with treatment. 
Similarly, others have also not found significant changes, before subgrouping, in indoleamine metabolites over the course of atypical antipsychotic treatment. ${ }^{78-80}$

In HC volunteers, Trp depletion may be associated with some mood lowering, some memory impairment and an increase in aggression. ${ }^{81}$ In patients with schizophrenia, Trp depletion exacerbated depressive symptoms and had no effects on positive or negative symptoms, ${ }^{82}$ whereas another study showed worsening negative symptoms. ${ }^{83}$ After antipsychotic treatment, however, Trp depletion may lead to an improvement on cognitive function including Wisconsin Card Sorting Test and Speed and Comprehension of Language Processing. ${ }^{84}$ In contrast, decreased levels of Mel may have a beneficial effect on memory formation. ${ }^{85}$

\section{Normal turnover of serotonin to 5-hydroxyindoleacetic} acid

Earlier, the role of serotonin in schizophrenia has been examined through 5HIAA, which is the principal metabolite of serotonin. A meta-analysis was carried out on the CSF, serum or urine levels of 5HIAA in patients with schizophrenia and HC volunteers from 12 studies, ${ }^{86}$ suggesting that 5HIAA levels were not altered in schizophrenia, whether in an acute phase or a chronic phase. Using CSF samples and HPLC/CEAS simultaneous measurements, Issa et $a .^{87}$ also found no differences in Trp pathway metabolites between schizophrenics and controls; however, NA5HT and Mel were not among the metabolites assayed. In this study, levels of 5HIAA were also not significantly different between HC and the two FENNS patient groups (Table 2), making it unlikely that the turnover of serotonin to 5HIAA is disturbed in schizophrenia. Lacking significant correlations between 5HIAA and Mel, kynurenine or 3-hydroxykynurenine in FENNS patient groups (Figure 5) are probably because of the fact that the levels of serotonin (precursor of 5HIAA) were also not significantly correlated with the above metabolites in the FENNS patient groups (Figure 4).

In contrast, significant within-group correlations between 5HT and Trp (Figure 4) and between 5HIAA and Trp (Figure 5) were shown only in the HC volunteers, but not in the FENNS patient groups (Figures 4 and 5). Moreover, the correlation between 5HIAA and Trp was significantly greater in the HC than the FENNS-BL groups (Figure 5). For a given dietary intake of Trp, there may be tighter regulatory control over 5HIAA production in the HC than in the FENNS group, significant when the latter patients have untreated psychosis (FENNS-BL). Although NA5HT and 5HIAA share the pathway precursors, Trp, 5HTP and serotonin, there is no change in 5HIAA reciprocal to the NA5HT increase in the FENNS-BL group, perhaps as there is a 20:1 ratio of 5HIAA to NA5HT produced. It is possible that the altered Trp5HIAA correlation and the NA5HT increase noted for this group are related.

\section{Kynurenine pathway}

Another branch of Trp catabolic cascade is the kynurenine pathway that produces neurotoxic (3-hydroxykynurenine and quinolinic acid) and neuroinhibitory (kynurenic acid) compounds (Figure 6). Kynurenic acid acts as a competitive antagonist of the glycine site of $N$-methyl-D-asparate receptors at high concentrations ${ }^{88}$ and as a noncompetitive antagonist of the $\alpha-7$-nicotinic acetylcholine receptor at a low concentration. ${ }^{89,90}$ Thus, increased levels of kynurenic acid may produce a spatial working memory dysfunction. ${ }^{90}$ Schwarcz et al..$^{26}$ have shown earlier increased cortical levels of kynurenic acid in schizophrenia, which may be related to cognitive impairment. ${ }^{91}$

Although plasma kynurenic acid was not determined in this study, we were unable to detect that levels of kynurenine and its metabolites, 3-hydroxykynurenine, anthranillic acid and 3-hydroxy anthranillic acid, and tryptamine, were significantly different between HC volunteers and two FENNS patient groups (Table 2). In addition, the ratio of kynurenine to Trp remained essentially the same among the three groups (Table 3) indicating that the enzyme indoleamine 2,3-dioxygenase was not altered in the FENNS patients. Subsequently, we searched for correlations between the levels of various metabolites to gain information about metabolic links. ${ }^{92}$ Like HC volunteers, both the FENNS patient groups also showed highly significant correlations between Trp and kynurenine, 3-hydroxykynurenine or tryptamine (Table 4). Moreover, each pair has an almost identical regression slope (data not shown) indicating tight control over relative metabolite ratios along the Trp pathways. It is likely that neither the kynurenine nor tryptamine pathways were altered in FENNS patients. After 24-h dietary intakes of Trp, Payne et al. ${ }^{48}$ have shown significant positive correlations between 5HIAA and kynurenic acid or xanthurenic acid in both schizophrenics and non-schizophrenics. Thus, these data support a notion that the same control mechanism may present in both HC and FENNS patients to regulate the levels of specific Trp metabolites through kynurenine and tryptamine pathways.

\section{Strengths and limitations}

Earlier studies in schizophrenia have been limited to one or a few metabolites in the Trp pathway. In this study, using a LCECA, we are able to measure $13 \operatorname{Trp}$ metabolites simultaneously in the plasma between FENNS and HC volunteers as well as between FENNS at $\mathrm{BL}$ and 4 weeks after antipsychotic treatment. The data collected from LCECA allow multiple rather than single metabolites to be used in markers for a group, which will greatly improve the predictive diagnostics for phenotypes that are directly involved in the neurotransmission and antioxidant defense system. More significantly, these comprehensive analyses that generate metabolic profiles represent not only biomarkers for disease, but also metabolic maps that can be used to identify specific genes responsible for disease. ${ }^{8,29,93}$ 
With regard to the covariate effects, the group differences in weight and height remained significant even after the significant gender effect was entered, raising the possibility of confounding these effects with 'group.' However, analyte dependence on covariates was minimal and not significant, so that any confounding of 'group' with covariates, in considering these effects on analytes, was considered unlikely.

The use of cross-validation to assess the performance of the logistic regression classifier tended for us to select smaller models than the traditional deviance-reduction method of inference. We note that this conservatism, plus some additional, is advisable as there is no unbiased estimator of the variance of prediction error (used in the error bars of Figure 3) as estimated by K-fold cross-validation. ${ }^{94}$ Ideally, one would have held out from analysis to this point some additional data samples for use in a completely independent test of our models and their parameters. Unfortunately, this was not possible with the limited data available, and an independent test must await collection of new data.

Regarding the correlations among analytes, it should be noted that explicit tests of differences in the non-null multiple correlations across the groups, using simultaneous Šidák 95\% confidence intervals (Larntz-Perlman), found a significant difference only for the Trp-5HIAA correlation. Hence, the differences in the detection of correlations significantly different from zero noted above for the three groups, contrasts with a general failure to detect the significance of correlation differences. The explanation may be either a lack of true differences or a failure to see true differences because of a larger variance when comparing two correlations with each other than when comparing one correlation with zero. Future research or larger numbers of samples would likely resolve this issue.

In this study, we have not carried out data analyses of biochemical correlations to clinical measures regarding symptom expression and illness severity because other related pathways and cross-pathway relationships are currently under investigation. To provide a better context, we will seek clustering of, and biological meaning in, group difference findings. This approach will seek clinical-biochemical associations by targeting key biochemical variables instead of pursuing large-scale multiple testing.

\section{Conflict of interest}

The authors declare no conflict of interest.

\section{Acknowledgments}

This material is based on work supported in part by the grants from the Department of Veterans Affairs, Veterans Health Administration, Office of Research and Development, Biomedical Laboratory R\&D (Merit Reviews (JKY) and Senior Research Career Scientist Award (JKY)), VA Pittsburgh Healthcare System (JKY,

GGD, RDR), National Institute of Health (MH58141 (JKY), MH64118 (RDR), $\quad$ MH45203 (MSK), R24 GM078233 (RKD), c UL1 RR024153 and NIH/NCRR/ GCRC Grant M01 RR00056), Metabolomics Research Network (RKD); Stanley Medical Research Institute (RKD), and NARSAD (RKD). The authors are grateful to Drs Nina Schooler, Cameron Carter and Gretchen Haas, and the clinical core staff of the Center for the Neuroscience of Mental Disorders (MH45156, David Lewis MD, Director) for their assistance in diagnostic and psychopathological assessments, and to P Cheng, C Korbanic and J Haflett for their technical assistance.

\section{References}

1 Lieberman JA, Koreen AR. Neurochemistry and neuroendocrinology of schizophrenia, a selective review. Schizophr Bull 1993; 19: 371-429.

2 Skosnik PD, Yao JK. From phospholipid and fatty acid defects to altered neurotransmission: is arachidonic acid a nexus in the pathophysiology of schizophrenia? Prostagland Leukot Essent Fatty Acids 2003; 69: 367-384.

3 Yao JK, van Kammen DP. Membrane phospholipids and cytokine interaction in schizophrenia. Int Rev Neurobiol 2004; 59: 297-326.

4 Javitt DC, Laruelle M. Neurochemical theories. In: Lieberman JA, Stroup TS, Perkins DO (eds). Text Book of Schizophrenia. American Psychiatric Publishing: Washington, DC, 2006, pp 85-116.

5 Tandon R, Keshavan MS, Nasrallah HA. Schizophrenia, 'Just the facts': what we know in 2008 Part 1: overview. Schizophr Res 2008; 100: 4-19.

6 Clish CB, Davidov E, Oresic M, Plasterer TN, Lavine G, Londo T et al. Integrative biological analysis of the APOE*3-leiden transgenic mouse. OMICS 2004; 8: 3-13.

7 Yao JK, Reddy RD. Metabolic investigation in psychiatric disorders. Mol Neurobiol 2005; 31: 193-203.

8 Kaddurah-Daouk R. Metabolic profiling of patients with schizophrenia. PLoS Med 2006; 3: e363.

9 Paige LA, Mitchell MW, Krishnan KR, Kaddurah-Daouk R, Steffens DC. A preliminary metabolomic analysis of older adults with and without depression. Int J Geriatr Psychiatry 2007; 22: 418-423.

10 Kaddurah-Daouk R, McEvoy J, Baillie RA, Lee D, Yao JK, Doraiswamy PM et al. Metabolomic mapping of atypical antipsychotic effects in schizophrenia. Mol Psychiatry 2007; 12: 934-945.

11 Kaddurah-Daouk R, Kristal BS, Weinshilboum RM. Metabolomics: a global biochemical approach to drug response and disease. Annu Rev Pharmacol Toxicol 2008; 48: 653-683.

12 Garelis E, Gillin J, Wyatt R, Neff N. Elevated blood serotonin concentration in unmedicated chronic schizophrenic patients. Am J Psychiatry 1975; 132: 184-186.

13 Stahl SM, Wood DJ, Mefford IN, Berger PA, Ciranello RD. Hyperserotoninemia and platelet serotonin uptake and release in schizophrenia and affective disorders. Am J Psychiatry 1983; 140: 26-30.

14 Jackman H, Luchins D, Meltzer HY. Platelet serotonin levels in schizophrenia: relationship to race and psychopathology. Biol Psychiatry 1983; 18: 887-902.

15 Muck-Seler D, Jakovljevie M, Deanovie Z. Time course of schizophrenia and platelet 5-HT level. Biol Psychiatry 1988; 23: 243-251.

16 Joseph M, Owen F, Baker H, Bourne R. Platelet serotonin concentration and monoamine oxidase activity in unmedicated chronic schizophrenic and schizoaffective patients. Psychol Med 1977; 7: 159-162.

17 Kolakowska T, Molyneux SG. Platelet serotonin concentration in schizophrenic patients. Am J Psychiatry 1987; 144: 232-234.

18 Ashcroft GW, Crawford TBB, Eccleston D, Sharman DF, MacDougall EG, Stanton JB et al. 5-Hydroxyindole compounds in the cerebrospinal fluid of patients with psychiatric or neurological disease. Lancet 1966; 2: 1049-1052.

19 Bowers MB. 5-Hydroxyindole acetic acid (5-HIAA) and homovanillic acid (HVA) following protenccid in acute psychiatric 
patients treated with phenothiazines. Psychopharmacology 1973; 28: 309-318.

20 Manowitz P, Gilmour DG, Racevskis J. Low plasma tryptophan levels in recently hospitalized schizophrenics. Biol Psychiatry 1973; 6: 109-118.

21 Domino EF, Krause RR. Free and bound serum tryptophan in drug-free normal controls and chronic schizophrenic patients. Biol Psychiatry 1974; 8: 265-279.

22 Wyatt RJ, Vaughan T, Galanter M, Kaplan J, Green R. Behavioral changes of chronic schizophrenic patients given L-5-hydroxytryptophan. Science 1972; 177: 1124-1126.

23 Gillin JC, Kaplan JA, Wyatt RJ. Clinical effects of tryptophan in chronic schizophrenic patients. Biol Psychiatry 1976; 11: 635-639.

24 Meltzer HY. Clinical studies on the mechanism of action of clozapine: The dopamine-serotonin hypothesis of schizophrenia. Psychopharmacology 1989; 99: S18-S27.

25 Meltzer HY. The mechanism of action of novel antipsychotic drugs. Schizophr Bulletin 1991; 17: 263-287.

26 Schwarcz R, Rassoulpour A, Wu H-Q, Medoff D, Tamminga CA, Roberts RC. Increased cortical kynurenate content in schizophrenia. Biol Psychiatry 2001; 50: 521-530.

27 Olney JW, Farber NB. Glutamate receptor dysfunction and schizophrenia. Arch Gen Psychiatry 1995; 52: 998-1007.

28 Yao JK, Cheng P. Determination of multiple redox-active compounds by high-performance liquid chromatography with coulometric multi-electrode array system. J Chromatogr $B$ 2004; 810: 93-100.

29 Kristal BS, Shurubor YI, Kaddurah-Daouk R, Matson WR. Highperformance liquid chromatography separations coupled with coulometric electrode array detectors: a unique approach to metabolomics. Methods Mol Biol 2007a; 358: 159-174.

30 Shi H, Vigneau-Callahan KE, Matson WR, Kristal BS. Attention to relative response across sequential electrodes improves quantitation of coulometric array. Anal Biochem 2002; 302: 239-245.

31 Shi H, Paolucci U, Vigneau-Callahan KE, Milbury PE, Matson WR, Kristal BS. Development of biomarkers based on diet-dependent metabolic serotypes: practical issues in development of expert system-based classification models in metabolomic studies. OMICS 2004; 8: 197-208.

32 Rozen S, Cudkowicz ME, Bogdanov M, Matson WR, Kristal BS, Beecher C et al. Metabolomic analysis and signature in motor neuron disease. Metabolomics 2005; 2: 101-108.

33 Bogdanov MB, Matson WR, Wang L, Matson T, Saunders-Pullman R, Bressman SS et al. Metabolomic profiling to develop blood biomarkers for Parkinson's disease. Brain 2008; 131: 389-396.

34 Schiavo S, Ebbel E, Sharma S, Matson W, Kristal BS, Hersch S et al. Metabolite identification using a nanoelectrospray LC-ECarray integrated system. Anal Chem 2008; 80: 5912-5923.

35 Eriksson L, Johansson E, Kettanah-Wold N, Wold S. Multi- and Megavariate Data Analysis. Umetrics AB: Malmo, Sweden, 2001.

36 Hatzimanikatis V, Li C, Ionita JA, Broadbelt LJ. Metabolic networks: enzyme function and metabolite structure. Curr Opin Struct Biol 2004; 14: 300-306.

37 Lehmann EL. Nonparametrics: Statistical Methods Based on Ranks. Holden and Day: San Francisco, p.20 (Rank Sum test) and p.130 (Signed Ranks Test) 1975.

38 Johnson RA, Wichern DW. Applied Multivariate Statistical Analysis, 4th edn, Prentice Hall: Upper Saddle River, NJ, USA, 1998, pp 193, 222-223.

39 Henze N, Zirkler B. A class of invariant consistent tests for multivariate normality. Commun Statist-Theory Meth 1990; 19 3595-3617.

40 Schott JR. Testing for complete independence in high dimensions. Biometrika 2005; 92: 951-956.

41 Larntz K, Perlman MD. A simple test for the equality of correlation matrices. In: Gupta SS, Berger JO (eds). Statistical Decision Theory and Related Topics IV, v. 2, Springer: New York, 1988, pp 289-298.

42 Drton M, Perlman MD. Model selection for Gaussian concentration graphs. Biometrika 2004; 91: 591-602.

43 Sidák Z. Rectangular confidence regions for the means of multivariate normal distributions. J Am Stat Assoc 1967; 62: 626-633.

44 Anderson TW. Introduction to Multivariate Statistical Analysis, 2nd edn, Wiley: New York, NY, USA, 1984, pp 102-155.
45 Kendall M, Gibbons JD. Rank Correlation Methods. Oxford University Press: New York, 1990, pp 60-69.

46 Hastie T, Tibshirani R, Friedman J. The Elements of Statistical Learning. Springer: New York, 2001a, pp 95-105.

47 Hastie T, Tibshirani R, Friedman J. The Elements of Statistical Learning. Springer: New York, 2001b, pp 193-217.

48 Payne IR, Walsh EM, Whittenburg EJR. Relationship of dietary tryptophan and niacin to tryptophan metabolism in schizophrenics and nonschizophrenics. Am J Clin Nutrition 1974; 27: 565-571.

49 Cooper JR, Bloom FE, Roth RH. Serotonin (5-hydroxytryptamine). In: Cooper JR, Bloom FE, Roth RH (eds). The Biochemical Basis of Neurophramacology, 4th edn. Oxford University Press: New York, 1982, pp 223-248.

50 Dubocovich ML, Masana MI. Melatonin receptor signaling. In: Encylopedia of Hormones. Elsevier Science: New York, NY, 2003, pp 638-643.

51 Brown GM, Pulido O, Grota LJ, Niles LP. N-Acetylserotonin in the central nervous system. Prog Neuropsychoparmacol Biol Psychiatry 1984; 8: 475-480.

52 Seithikurippu RP-P, Trakht I, Venkataramanujan S, Spence DW, Maestroni GJM, Zisapel $\mathrm{N}$ et al. Physiological effects of melatonin: role of melatonin receptors and signal transduction pathways. Prog Neurobiol 2008; 85: 335-353.

53 Skinner DC, Malpaux B. High melatonin concentrations in third ventricular cerebrospinal fluid are not due to galen vein blood recirculating through the choroid plexus. Endocrinology 1999; 140: 4399-4405.

54 Tricoire H, Locatelli A, Chemineau P, Malpaux B. Melatonin enters the cerebrospinal fluid through the pineal recess. Endocrinology 2002; 143: 84-90.

55 Reiter RJ. Melatonin: clinical relevance. Best Pract Res Clin Endocrinol Metab 2003; 17: 273-285.

56 Ernster I. Lipid peroxidation in biological membranes: mechanisms and implications. In: Yagi K (ed). Active Oxygen Species, Lipid Peroxides, and Antioxidants. CRC Press: Tokyo, Japan, 1993, pp 11-38.

57 Whatley SA, Curti D, Das Gupta F, Ferrier IN, Jones S, Taylor C et al. Superoxide, neuroleptics and the ubiquinone and cytochrome b5 reductases in brain and lymphocytes from normals and schizophrenic patients. Mol Psychiatry 1998; 3: 227-237.

58 Yao JK, Reddy RD, van Kammen DP. Oxidative damage and schizophrenia: an overview of the evidence and its therapeutic implications. CNS Drugs 2001; 15: 287-310.

59 Mahadik SP, Parikh V, Khan MM. The role of oxidative stress in modulating membrane and phospholipid function in schizophrenia. In: Peet M, Glen I, Horrobin D (eds). Phospholipid Spectrum Disorders in Psychiatry and Neurology. Marius Press: Carnforth, UK, 2003, pp 277-288.

60 Ben-Shachar D, Laifenfeld D. Mitochondria, synaptic plasticity, and schizophrenia. Int Rev Neurobiol 2004; 59: 273-296.

61 Bubber P, Tang J, Haroutunian V, Xu H, Davis KL, Blass JP et al. Mitochondrial enzymes in schizophrenia. J Mol Neurosci 2004; 24: 315-321.

62 Yao JK, Leonard S, Reddy RD. Altered glutathione redox state in schizophrenia. Dis Markers 2006; 22: 83-93.

63 Gysin R, Kraftsik R, Sandell J, Bovet P, Chappuis C, Conus P et al. Impaired glutathione synthesis in schizophrenia: Convergent genetic and functional evidence. Proc Natl Acad Sci USA 2007; 104: 16621-16626.

64 Poeggeler B, Reiter RJ, Tan DX, Chen LD, Manchester LC. Melatonin, hydroxyl radical-mediated oxidative damage, and aging: a hypothesis. J Pineal Res 1993; 14: 151-168.

65 Tan DX, Reiter RJ, Manchester LC, Yan MT, El-Sawi M, Sainz RM et al. Chemical and physical properties and potential mechanisms: melatonin as a broad spectrum antioxidant and free radical scavenger. Curr Top Med Chem 2002; 2: 181-197.

66 Marshall KA, Reiter RJ, Poeggeler B, Aruoma OI, Halliwell B. Evaluation of the antioxidant activity of melatonin in vitro. Free Radic Biol Med 1996; 21: 307-315.

67 Barlow-Walden LR, Reiter RJ, Abe M, Pablos M, Menendez-Pelaez A, Chen LD et al. Melatonin stimulates brain glutathione peroxidase activity. Neurochem Int 1995; 26: 497-502.

68 Urata Y, Honma S, Goto S, Todoroki S, Iida T, Cho S et al. Melatonin induces gamma-glutamylcysteine synthetase mediated 
by activator protein-1 in human vascular endothelial cells. Free Radic Biol Med 1999; 27: 838-847.

69 Antolin I, Rodriguez C, Sainz RM, Mayo JC, Uria H, Kotler ML et al. Neurohormone melatonin prevents cell damage: effect on gene expression for antioxidant enzymes. FASEB J 1996; 10: 882-890.

70 Longoni B, Pryor WA, Marchiafava P. Inhibition of lipid peroxidation by $N$-acetylserotonin and its role in retinal physiology. Biochem Biophys Res Commun 1997; 233: 778-780.

71 Garcia JJ, Reiter RJ, Guerrero JM, Escames G, Yu BP, Oh CS et al. Melatonin prevents changes in microsomal membrane fluidity during induced lipid peroxidation. FEBS Lett 1997; 408: 297-300.

72 Garcia JJ, Reiter RJ, Karbownik M, Calvo JR, Ortiz GG, Tan DX et al. $\mathrm{N}$-acetylserotonin suppresses hepatic microsomal membrane rigidity associated with lipid peroxidation. Eur J Pharmacol 2001; 428: 169-175.

73 Duell PB, Wheaton DL, Shultz A, Nguyen H. Inhibition of LDL oxidation by melatonin requires supraphysiologic concentrations. Clin. Chem 1998; 44: 1931-1936.

74 Wolfler A, Abuja PM, Schauenstein K, Liebmann PM. N-acetylserotonin is a better extra- and intracellular antioxidant than melatonin. FEBS Lett 1999; 449: 206-210.

75 Wang HX, Liu F, Ng TB. Examination of pineal indoles and 6-methoxy-2-benzoxazolinone for antioxidant and antimicrobialeffects. Comp Biochem Physiol C Toxicol Pharmacol 2001; 130: 379-388.

76 Fagali N, Catala A. The effect of melatonin and structural analogues on the lipid peroxidation of triglycerides enriched in omega-3 polyunsaturated fatty acids. Life Sci 2007; 81: 299-305.

77 Gavazza MB, Català A. Protective effect of N-acetyl-serotonin on the nonenzymatic lipid peroxidation in rat testicular microsomes and mitochondria. J Pineal Res 2004; 37: 153-160.

78 van der Heijdena FMMA, Tuiniera S, Fekkesb D, Sijbena AES, Kahnc RS, Verhoevena WMA. Atypical antipsychotics and the relevance of glutamate and serotonin. Eur Neuropsychopharmacol 2004; 14: 259-265.

79 Scheepers FE, Gispen-de Wied CC, Westenberg HGM, Kahn RS. The effect of olanzapine treatment on monoamine metabolite concentrations in the cerebrospinal fluid of schizophrenic patients. Neuropsychopharmacology 2001; 25: 468-475.

80 Sailer CF, Salama AI. Seroquel: biochemical profile of a potential atypical antipsychotic. Psychopharmacology 1993; 112: 285-292.

81 Bell C, Abrams J, Nutt D. Tryptophan depletion and its implications for psychiatry. Br J Psychiatry 2001; 178: 399-405.
82 D’Souza D, Gil R, Abi-Draghjam A. Tryptophan depletion paradigm: implications for schizophrenia. Biol Psychiatry 1994; 35: 703.

83 Sharma RP, Shapiro LE, Kamath SK, Soll EA, Watanabe MD, Davis JM. Acute dietary tryptophan depletion: effects on schizophrenic positive and negative symptoms. Neuropsychobiology 1997; 35: 5-10.

84 Golightly K, Lloyd J, Hobson J, Gallagher P, Mercer G, Young AH. Acute tryptophan depletion in schizophrenia. Psychol Med 2001; 31: 75-84.

85 Rawashdeh O, de Borsetti NH, Roman G, Cahill GM. Melatonin suppresses nighttime memory formation in Zebrafish. Science 2007; 318: 1144-1146.

86 Tuckwell HC, Koziol JA. On the concentration of 5-hydroxyindoleacetic acid in schizophrenia: a meta-analysis. Psychiatry Res 1996; 59: 239-244.

87 Issa F, Gerhardt GA, Bartko JJ, Suddath RL, Lynch M, Gamache PH et al. A multidimensional approach to analysis of cerebrospinal fluid biogenic amines in schizophrenia: I. Comparisons with healthy control subjects and neuroleptic-treated/unmedicated pairs analyses. Psychiatry Res 1994; 52: 237-249.

88 Stone TW. Neuropharmacology of quinolinic and kynurenic acids. Pharmacol Rev 1993; 45: 309-379.

89 Hilmas C, Pereira EFR, Alkondon M, Rassoulpour A, Schwarcz R, Albuquerque EX. The brain metabolite kynurenic acid inhibits $\alpha 7$ nicotinic receptor activity and increases non- $\alpha 7$ nicotinic receptor expression: physiopathological implications. J Neurosci 2001; 21: 7463-7473.

90 Alkondon M, Pereira EFR, Yu P, Arruda EZ, Almeida LEF, Guidetti $\mathrm{P}$ et al. Targeted deletion of the kynurenine aminotransferase II gene reveals a critical role of endogenous kynurenic acid in the regulation of synaptic transmission via $\alpha 7$ nicotinic receptors in the hippocampus. J Neurosci 2004; 24: 4635-4648.

91 Chess AC, Simoni MK, Alling TE, Bucci DJ. Elevations of endogenous kynurenic acid produce spatial working memory deficits. Schizophr Bulletin 2007; 33: 797-804.

92 Kose F, Weckwerth W, Linke T, Fiehn O. Visualization of plant metabolomic correlation networks using clique-metabolite matrices. Bioinformatics 2001; 17: 1198-1208.

93 Watkins SM. Comprehensive lipid analysis: a powerful metanomic tool for predictive and diagnostic medicine. Isr Med Assoc J 2000; 2: 722-724.

94 Bengio Y, Grandvalet Y. No unbiased estimator of the variance of K-fold cross-validation. J Mach Learn Res 2004; 5: 1089-1105. 